6

\title{
MicroRNA-transcriptome networks in whole blood and monocytes of women undergoing preterm labor
}

Alison G. Paquette, $\mathrm{PhD}^{1}$; Oksana Shynlova, $\mathrm{PhD}^{2}$; Xiaogang $\mathrm{Wu}, \mathrm{PhD}^{1}$; Mark

Kibschull, $\mathrm{PhD}^{2}$; Kai Wang, $\mathrm{PhD}^{1}$; Nathan D. Price, $\mathrm{PhD}^{1}$; Stephen J Lye, $\mathrm{PhD}^{2 *}$

1. Institute for Systems Biology, Seattle WA 98119 2. Program in Development and Fetal Health, Lunenfeld-Tanenbaum Research Institute, Sinai Health System, Toronto, Ontario, Canada

*Corresponding Author: Dr Stephen J Lye

Address: Lunenfeld-Tanenbaum Research Institute at Sinai Health System 6-1004 - 25 Orde Street, Toronto, Ontario, M5T 3H7

Tel: 416-558-8640

Email: lye@lunenfeld.ca 


\section{ABSTRACT}

19 Preterm birth is attributed to neonatal morbidity as well as cognitive and physiological

20 challenges. We have previously identified significant differences in mRNA expression in

21 whole blood and monocytes, as well as differences in miRNA concentration in blood

22 plasma, extracellular vesicles (EV) and EV-depleted plasma in women undergoing

23 spontaneous preterm labor (sPTL). The goal of this analysis was to identify differences in

24 miRNA expression within whole blood (WB) and peripheral monocytes (PM) from the

25 same population of women undergoing sPTL compared to nonlaboring controls matched

26 by gestational age. We performed single end small RNA sequencing in whole blood and

27 peripheral monocytes from women undergoing sPTL with active contractions(24-34

28 weeks of gestation, $\mathrm{N}=15$ ) matched for gestational age to healthy pregnant non laboring

29 controls (>37 weeks gestation, $\mathrm{N}=30$ ) who later delivered at term as a part of the Ontario

30 Birth Study (Toronto, Ontario CA). We identified significant differences in expression of

3116 miRNAs in PMs and 9 miRNAs in WB in women undergoing sPTL. In PMs, these

32 miRNAs were predicted targets of 541 genes, including 28 previously associated with

33 sPTL. In WB, miRNAs were predicted to target 303 genes, including 9 previously

34 associated with sPTL. These genes were involved in a variety of immune pathways,

35 including interleukin 2 signaling. This study is the first to identify changes in miRNA

36 expression in WB and PMs of women undergoing sPTL. Our results shed light on

37 potential mechanisms by which miRNAs may play a role in mediating systemic

38 inflammatory response in pregnant women that deliver prematurely. 
Premature birth, defined as delivery before 37 weeks of gestation, occurs in

$41 \quad 11.1 \%$ of pregnancies worldwide and is associated with neonatal morbidity and

42 mortality[1]. Pregnancies characterized by pathological changes including placental

43 insufficiency, subclinical infections, disruptions in maternal immune-tolerance to

44 pregnancy, and decidual senescence often result in spontaneous preterm labor (sPTL).

45 There is a paucity of research examining transcriptomic changes that occur during

46 sPTL[2], which have the potential to transform our understanding of the molecular

47 mechanisms underlying this heterogeneous syndrome [3] . Therefore, there is a crucial

48 research need for robust and multidimensional characterization of transcriptomic changes

49 relating to $\mathrm{SPTL}$.

51 environment and responds to fetal cues. At the beginning of gestation, the number of

52 monocytes in maternal blood drastically increase[4], which regulate placental invasion,

53 angiogenesis, and tissue remodeling [5]. After infiltration into uterine tissues, monocytes

54 differentiate into macrophages characterized by an immunosuppressive (M2) phenotype

55 in normal pregnancies and an inflammatory (M1) state in complicated pregnancies[5] .

56 Maternal immune cells play a crucial role in normal pregnancy maintenance and provide

57 insight into changes that occur in pregnancies complicated by preterm birth.

59 transcriptional regulators of gene expression in all human organs. MiRNAs are expressed

60 within the placenta throughout pregnancy and are involved in fetal and maternal

61 signaling. Placental miRNA expression profiles have been associated with preeclampsia

$62[6]$ [7] and sPTL[8]. Like other organs, the placenta releases miRNAs into the circulation, 
and unique placenta derived miRNAs (from the C19MC and C14MC miRAN cluster) are detectable within maternal plasma[9-11]. Differential miRNA expression in plasma has been detected in pregnancies complicated by fetal growth restriction preeclampsia[13], and preterm birth $[14,15]$. Circulating placenta enriched miRNAs are detectable in whole blood, and have been associated with fetal hypoxia[16]. MiRNAs may also play a role in monocyte differentiation throughout pregnancy, as there are differences in miRNA concentration in different monocyte subpopulations (CD16+ vs. CD16-)[17]. miRNAs have emerged as important transcriptional regulators and signaling molecules during pregnancy with the potential to play a role in the underlying molecular perturbations that occur during pregnancy-related complications such as sPTL.

Genome scale transcriptomic analysis of maternal blood provides a window into the changes that occur throughout pregnancy and during parturition. We have previously identified differences in mRNA expression between whole blood and peripheral monocytes[18], as well as differences in miRNA concentration in whole plasma, extracellular vesicles (EVs), and EV depleted plasma in women undergoing sPTL. The goal for this analysis was to identify changes in miRNA expression in maternal whole blood and monocytes in the same population (15 women undergoing sPTL and 30 pregnant women matched on gestational age not undergoing labor) and to integrate these results with our previous findings. We hypothesize that there are changes in miRNAs in whole blood and monocytes of women undergoing sPTL compared to controls, which are related to the role of these miRNAs in transcriptional regulation of labor-associated genes. Through this miRNA analysis and integration with next generation sequencing 
mRNA data from the same individuals, we obtain a better understanding of the transcriptional regulation that occurs in the context of PTL.

\section{MATERIALS AND METHODS}

\section{Study Design}

Participants were recruited within the Ontario Birth Study (OBS); a continuously enrolling prospective cohort at Mount Sinai Hospital (MSH) in Toronto Canada.

Inclusion criteria for the OBS are: pregnancy diagnosed $<17$ weeks, maternal age $>18$, English speaking, signed informed consent, and intent to deliver and receive antenatal care at MSH. Exclusion criteria included non-viable neonate and/or inability to provide consent. Peripheral blood was collected from 15 patients who were in preterm labor, defined as cervical dilatation $>4 \mathrm{~cm}$ and active uterine contractions, with no other accompanying pathology; who delivered prematurely between 24-34 weeks of gestation (Preterm labor or PTL). They were matched with 30 healthy asymptomatic pregnant women whose blood was taken at the same time point during routine clinical visits(gestational age 24-34 weeks), who later delivered at full term (TL). A full description of this subpopulation is described in other manuscripts [18] [19]. This study was approved by the Research Ethics Board of Mount Sinai Hospital, Toronto, Canada (\#04-0024-E). All patients provided written consent as part of the OBS at Sinai Health System, Toronto, Canada.

\section{Specimen Collection}

In PTL patients, peripheral blood samples were collected prospectively at the point of hospital admission, and in TL controls blood was collected during the regular antenatal 
107 visit. Blood was collected into both PAXgene tubes (Qiagen; Hilden, Germany) for

108 whole blood isolation and EDTA blood collection tubes for monocyte separation.

109 Monocytes were separated through the Monocyte RosetteSep cocktail (Stemcell

110 Technologies, Vancouver CA), followed by high density gradient centrifugation to

111 generate a highly purified monocyte fraction for subsequent mRNA isolation.

\section{Small RNA Sequencing and Quantification}

113 Whole blood RNA was isolated using a PAXgene blood miRNA kit (Qiagen Hilden,

114 Germany), and from peripheral monocytes using TRIzol LS reagent (Thermo Fisher,

115 Waltham MA) following manufacturer's instructions. RNA quality was determined using

116 Experion analyzers (BioRad, Hercules CA) and sequenced at The Center for Applied

117 Genomics at the SickKids Hospital, Toronto, Canada. Library preparation was performed

118 following the New England Biolabs NEB Next multiplex small library preparation

119 protocol, with $400 \mathrm{ng}$ of total RNA as the input. The 3' adapter was ligated to the small

120 RNA, followed by reverse transcriptase (RT) primer hybridization to the 3' adapter and

121 then the 5' adapter was ligated to the opposite end. Libraries were generated using first

122 stranded synthesis then enriched by PCR. Quality and size was determined using the

123 Bioanalyzer 2100 DNA High Sensitivity chip (Agilent Technologies, Palo Alto CA), and

124 libraries were quantified by qPCR using the Kapa Library Quantification Illumina/ABI

125 Prism Kit protocol (KAPA Biosystems, Wilmington MA) and sequenced on Illumina

126 HiSeq 2500 (Illumina, San Diego CA). 
129 sequences were trimmed and low-quality sequences were removed. Processed sequences

130 were aligned to all human miRNAs (miRBase Release 21,[21] ) with no mismatches. We

131 removed miRNAs with 0 read counts in $>50 \%$ of samples or a mean read count $<20$

132 producing a final dataset of 417 miRNAs in whole blood and 274 miRNAs in peripheral

133 monocytes, CPM (Count Per Million) values were calculated using RNA sequencing

134 analysis software "edgeR"[22], and $\log 2$ transformed. Data from this analysis is publicly

135 available within the Gene Expression Omnibus (GEO) as GSE108876 and GSE108877.

\section{Statistical Analyses}

137 Differentially expressed miRNAs were identified using edgeR [22]. miRNAs were

138 considering significantly associated with PTL if they exhibited a Benjamini Hochberg

139 (BH) adjusted $q$ value of $<0.05$, and a $\log 2$ fold change of $>1$. In order to mitigate batch

140 effects, we eliminated differentially expressed miRNAs which were correlated with

141 order they were loaded onto the array ( $\mathrm{N}=3$ miRNAs in whole blood and monocytes),

142 identified using spearman correlation tests with a statistical cutoff of FDR adjusted $q$

143 value $<0.05$. We compared expression of miRNA in whole blood and peripheral

144 monocytes to previously published data from the same patient population, including

145 miRNA data from matched plasma and EVs(GSE106224)[19], as well as mRNA

146 expression from whole blood and peripheral monocytes (GSE96097) [18].

147 Putative miRNA targets were detected using the quantitative model TargetScan (V. 7.0,

148 targetscan.org), which characterizes canonical targeting of miRNAs based on 14 features,

149 and has the best predictive performance compared to comparable tools[23]. MiRNA-

150 target gene relationships were identified in whole blood and peripheral monocytes by

151 examining correlations between each miRNA and its proposed target genes. For each 
152 miRNA, we used only mRNA targets with an absolute value of "context++" score (a

153 metric of miRNA target prediction accuracy used by TargetScan) which was higher than

154 the median, ensuring only highest quality relationships were validated. miRNA and target

155 relationships were considered statistically significant using a Spearman correlation

156 coefficient $<-0.3$ and a $p<0.05$. These mRNAs were then matched to the list of

157 Differentially Expressed Genes (DEGs) identified in whole blood and monocytes from

158 the same individuals [18].

Gene set enrichment analysis was performed for mRNA targets using two-sided

160 hypergeometric tests conducted on Gene Ontology (GO) Biological Process Gene sets using GO gene set visualization application "ClueGO" within the cytoscape environment [24]. GO gene sets with more than 5 genes were included in the analysis, and were

163 considered significant with a Benjamini Hochberg adjusted $q<0.05$. These GO gene sets 164 were clustered into ClueGO groups based on the similarity in the number of genes in the 165 gene sets calculated by a Kappa score within ClueGO. Data was analyzed and visualized 166 in R (Version 3.3.1) and Cytoscape (Version 3.6.0) [24].

\section{RESULTS}

170 uterine contractions with cervical dilation followed by delivery between 24-34 weeks)

171 were compared to healthy women not in labor that went on to deliver at full term (Table

172 1). There were no significant differences in delivery method, ethnicity, or fetal sex

173 between sPTL cases and controls ( $p>0.05$, Fisher's exact test). Women who delivered 
174 prematurely were more likely to be between 18 and 30 years old vs. older than 30 ,

175 although this relationship was borderline statistically significant $(p=0.06)$.

\section{Differences in miRNA expression in Whole Blood and Monocytes}

We identified miRNAs with significantly different concentrations in peripheral monocytes and whole blood of women undergoing PTL using generalized linear models within edgeR [22]. In monocytes, 11 miRNAs were higher and 5 miRNAs were lower in women undergoing PTL compared to women who delivered at term (Figure 1A-B, supplemental table 1). In whole blood, 6 miRNAs were higher and 3 miRNAs were lower in women undergoing PTL compared to controls (Figure 1 C-D, supplemental

table 1). Two of these miRNAs (mIR-495-3p and miR-381-3p) were from the C14MC

MC miRNA cluster; a region of chromosome 14 which encodes miRNAs which are substantially enriched in placental tissue. Overall, there was a higher number of significant miRNAs and larger concentration changes in relation to SPTL in monocytes compared to whole blood.

MiR-1291-5p expression was significantly increased in whole blood of women undergoing sPTL(Log fold change 1.12, FDR adjusted $\left.q=2.22 \times 10^{-4}\right)$, but decreased in monocytes of women undergoing sPTL (Log fold change -1.32, FDR adjusted $\left.q=2.45 \times 10^{4}\right)$. We observed a significant negative correlation between expression of this miRNA in whole blood and in monocytes $(p=0.02, \rho=-0.02$, Spearman correlations,

194 populations of blood cells (such as erythrocytes, platelets, lymphocytes or granulocytes) 
195 may have the opposite expression profile compared to monocytes and also contribute to

196 the miRNA pool in women undergoing sPTL.

\section{Comparison of expression differences across blood compartments}

199 identified significant differences in concentration of 132 miRNAs in whole plasma, EVs

200 and EV depleted plasma [19]. Five of these miRNAs were also significantly different in

201 monocytes, and three miRNAs were significantly different in whole blood

202 (supplemental table 2). We examined correlations of these 8 miRNAs that were

203 significantly upregulated in plasma samples as well as in whole blood and monocytes

204 from the same patients (supplemental figure 2, N=44 samples with plasma and whole

205 blood RNAseq data, N=42 samples with monocyte and whole blood sRNA sequencing

206 data, and N=14 samples with RNAseq data in EVs and monocytes/whole blood).

208 (miR-328-3p) was decreased in both plasma and monocytes of women undergoing sPTL

209 compared to TL controls. The concentration/expression of these miRNAs was not

210 significantly correlated between whole plasma and monocytes $(p>0.05$, Spearman

211 correlations). MiR-181b-5p was increased in monocytes and decreased in the plasma of

212 women undergoing sPTL, and the expression/concentration of these miRNAs was

213 inversely correlated in plasma and monocytes $(\rho=-0.28, p=0.07)$. MiR-378c-5p was

214 increased in both monocytes and in extracellular vesicles of women undergoing sPTL.

215 We observed significant positive correlations $(p<0.05)$ between whole blood miRNA

216 levels and the levels in plasma and EVs in the 3 miRNAs significantly associated with 
217 sPTL in the previous studies: (1) MiR-374a-5p was decreased in both plasma and whole

218 blood of women undergoing sPTL, (2) miR-381-3p was decreased in plasma but

219 increased in whole blood of women undergoing sPTL, and (3) miR495-5p was decreased

220 in the plasma and extracellular vesicles but increased in the whole blood of women

221 undergoing sPTL compared to controls. This indicates that there is some overlap in signal

222 related to sPTL that is detectable across different components of the blood within the

223 same individuals.

224 Confirmation of target mRNAs of miRNAs associated with sPTL

We identified the putative mRNA targets of the differentially expressed miRNAs

226 using the TargetScan database. TargetScan incorporates 14 features to predict miRNA-

227 mRNA interactions and has shown to have the highest predictive value compared to

228 similar tools [23]. From the TargetScan database, we included only the top 50\% of

229 predicted mRNA based on the "context ++" score (a metric of accuracy used by the

230 database). We then compared mRNA expression of these predicted targets to the miRNA

231 concentration within matched samples (GSE96097,[18] ). We constrained miRNA-

232 mRNA predicted interactions to only those which had a Spearman correlation coefficent

$233 \rho<-0.3$ and a $p<0.05$, which represented $7.29 \%$ of the predicted genes in monocytes and

234 4.26\% in whole blood (Supplemental table 3, Supplemental Figure 4). In monocytes,

235541 genes were associated with 16 miRNAs, and in whole blood 303 genes were

236 associated with 7 miRNAs. Based on this criteria, there were 2 miRNAs in whole blood

237 with no confirmed mRNA targets. 
240 Here, we compared the changes in mRNA expression related to sPTL and the confirmed

241 miRNA target genes we have identified in this current study (Figure 2). In whole blood,

2429 of the 181 DEGs were confirmed miRNA targets of 3 differentially expressed miRNAs

243 in whole blood (Shown in Figure 2A). Among these 3 miRNAs in whole blood, miR-

$244 \quad 4742-3 p$ emerged as the strongest regulator, since it was negatively associated with 5

245 genes which were positively associated with sPTL, including $S P H, C D 177, C Y P 1 B 1$,

246 ELOVL7 and GRB10. In monocytes, 28 of these 262 genes were confirmed mRNA

247 targets of 7 differentially expressed miRNAs. In monocytes, MiR-1291-5p emerged as

248 the strongest regulator, and was negatively associated with 11 of the 28 genes which are

249 positively associated with sPTL, including $I L 1 B, I L 1 R I$ and $C D 177$ (Figure $2 B$ ). In both

250 whole blood and monocytes, the direction of the associations with PTL is congruent with

251 the directionality in mRNAs (i.e., if a miRNA is positively associated with PTL, the

252 miRNA is negatively correlated with mRNAs that are negatively associated with PTL).

253 We also examined mRNAs which were in the same genomic region as the miRNAs in

254 our study, but found that none of the associated mRNAs were statistically significantly

255 associated with sPTL in monocytes and whole blood leukocytes. This integrated analysis

256 suggests that the miRNAs identified here may play a role in the transcriptional regulation

257 of genes associated with SPTL in monocytes and whole blood leukocytes.

\section{$258 \quad$ miRNA target mRNA networks in sPTL}


261 identified in whole blood and monocytes. Using CytoScape application "ClueGO”, we

262 identified significantly enriched GO gene sets which were grouped together based on

263 intersecting common genes, which are shown in Figure 3, and in supplemental table 4.

264 In whole blood, 59 different GO gene sets were significantly enriched for the 303 genes

265 which were mRNA targets for the 9 miRNAs associated with sPTL in whole blood.

266 These gene sets were grouped into 19 ClueGO groups, including 8 distinct groups (i.e.

267 only had $1 \mathrm{GO}$ gene set), and one ClueGO group which contained $22 \mathrm{GO}$ gene sets

268 including the 5 most significant gene sets: "positive regulation of cytokine production" ,

269 "positive regulation of adaptive immune response" , "interleukin-2 production",

270 "positive regulation of adaptive immune response based on somatic recombination of

271 immune receptors built from immunoglobulin superfamily domains”, and" $\mathrm{T}$ cell

272 receptor signaling pathway". This diversity of GO gene sets and strong signal indicates

273 that these miRNAs associated with sPTL are involved in a wide variety of biological

274 pathways in whole blood; particularly immune pathways.

276 which were targets for the 16 miRNAs associated with sPTL. These gene sets were

277 clustered into 11 groups, including 8 gene sets which were distinct (i.e only had 1 GO

278 term), and 1 gene set (Group 10), which had 13 different terms. The top 5 GO terms

279 based on q value were: "small GTPase mediated signal transduction"," negative

280 regulation of protein modification process", "positive regulation of interleukin 2

281 production", "positive regulation of mononuclear cell proliferation", and "negative

282 regulation of protein kinase activity". These terms are reflective of changes associated 
283 with monocyte proliferation and immune function, indicating that miRNAs may play a

284 role in regulation of these pathways in monocytes.

The Gene Ontology gene set "positive regulation of interlukin-2 production” was

286 significantly enriched in both whole blood and monocytes. This gene set contains 31

287 genes, and the differentially expressed miRNAs in both whole blood and monocytes were

288 predicted negative regulators of 5 unique genes within this gene set, each representing

$28917.86 \%$ of the total genes in this pathway. In whole blood, 4 miRNAs were identified as

290 regulators of the 5 genes (CCR2, CD4, MALT1, PDE4B, and CD28). In monocytes, 3

291 miRNAs were identified as negative regulators of the 5 genes ( $A S H 3, C D 83, I L 1 B$,

292 TNFSF4, and CD28) involved in interleukin signaling (Table 2). In this gene set, IL1B

293 was a target of miR-1291-5p, and IL1B expression was significantly increased in women

294 undergoing sPTL (FDR adjusted $q=4.02 \times 10-3, \log \mathrm{FC}=2.01$ ). Through this analysis, we

295 have identified that miRNA expression within whole blood and peripheral monocytes

296 significantly alters genes involved in production of interleukin 2.

\section{DISCUSSION}

This study is the first comprehensive miRNA profiling of women undergoing

299 sPTL using small RNA sequencing within both whole blood and peripheral monocytes.

300 Our key findings included (A) identification of differentially expressed miRNAs in whole

301 blood and peripheral monocytes, which (B) were negative regulators of genes associated

302 with SPTL, which we identified using previously generated RNA sequencing data on

303 whole blood and monocytes from the same individuals and (C) identification of unique 
304 and shared gene sets enriched for mRNA targets of these differential miRNAs in

305 monocytes and whole blood of women undergoing sPTL.

Overall, we identified a greater number of miRNAs with concentration changes, and more genes related to miRNAs associated with sPTL. Additionally, we observed no

308 congruence in the differential miRNAs identified in monocytes and whole blood.

309 Maternal blood includes three major subsets of immune cells; monocytes, lymphocytes,

310 and granulocytes, which circulate through gestational tissues including the myometrium,

311 decidua and placenta, and are exposed and respond to signals from these tissues. RNA

312 sequencing data derived from monocytes exhibits less cellular heterogeneity and thus

313 likely has a cleaner signal. We suggest that the signaling we observe in monocytes may

314 be related to their functional role throughout pregnancy and parturition [4] . Altogether,

315 our work suggests that a stronger signal related to sPTL can be obtained from monocytes 316 compared to whole blood.

We observed a number of miRNAs which were differentially expressed in in

318 whole blood and monocytes of women undergoing sPTL which also exhibited significant

319 concentration differences previously identified in whole plasma, EV-depleted plasma and

320 EVs in the same population of individuals [19]. We identified fewer differential miRNAs

321 in immune cells compared to the plasma component, but overlap in the differentially

322 expressed miRNAs, as well as concurrence in the directionality of associations with

323 sPTL. This suggests functional signaling that occurs within blood in context of sPTL. In

324 independent populations, increased expression of miR-223 has been identified in plasma

325 of women at 20 weeks who went on to deliver prematurely[14]. Similarly, we observed

326 increased levels of this miRNA in whole blood of women who delivered prematurely 
327 compared to TL controls, which supports the potential of this miRNA as having a

328 functional role in PTL.

In whole blood, 2 of the miRNAs (miR-495-3p and miR-381-3p) with concentration differences in women undergoing sPTL were part of the C14MC region; a

331 group of imprinted miRNAs located on chromosome 14q32 which is highly produced by

332 the placenta and enters maternal circulation [25]. In plasma samples from the same

333 patients, these miRNA concentrations were negatively associated with sPTL[19], and

334 changes in average expression of C14MC miRNAs in plasma have also been associated

335 with preterm labor[15]. On average, C14MC miRNAs have higher expression and lower

336 variance in the fetal compartment and the placenta compared to the maternal plasma, thus

337 this signal may be reflecting changes in this compartment[26]. We hypothesize that the

338 changes observed within the whole blood and monocyte fraction of leukocytes are

339 reflective of functional transcriptional changes that occur within the blood or they may be

340 reflective of concentration changes related to miRNA signals from the developing fetus.

We identified mRNAs which were transcriptionally regulated by differentially

342 expressed miRNAs using predicted targets from TargetScan, which were filtered using

343 matched mRNA data derived through RNA sequencing based on negative correlations

344 with miRNA[18]. Of the targets identified by TargetScan, on average only $4.26 \%$ of

345 these predicted targets passed our filter whole blood and 7.29\% in monocytes. These

346 target prediction algorithms are only designed to reveal gene expression regulation

347 potential, and expression can also be altered by post transcriptional modifications

348 including transcriptional regulation, histone acetylation and DNA methylation [27], and

349 are not cell type specific [23]. This analysis was designed to collect miRNA and mRNA 
350 from the same patient, which allowed us to perform correlations and obtain a far more

351 accurate understanding of miRNA-mediated regulation of gene expression than by

352 relying solely on miRNA prediction algorithms alone.

We used our curated, confirmed miRNA target lists generated to perform an

354 enrichment analysis of gene sets related to our differentially expressed miRNAs. In

355 whole blood, we identified immune related gene sets. This may be reflective of the

356 inflammatory and immune response that occurs as a breakdown in maternal fetal

357 tolerance or inflammation related to preterm labor[28]. The target genes of the miRNAs

358 differentially expressed within monocytes were enriched for a total of 19 GO gene sets

359 including those related to interleukin production and activation of the immune system,

360 such as "regulation of interleukin production (Interleukins 2, 4 and 6), as well as T Cell,

361 lymphocyte, and monocyte proliferation. The monocyte population expands during

362 pregnancy and differentiates into a pro-inflammatory phenotype during labor [30]. Our

363 work indicates that part of this expansion in monocytes may be transcriptionally

364 regulated by a subset of miRNAs in monocytes. More work is required to understand if

365 these miRNAs induce monocyte expansion and proliferation in vitro, which is beyond the

366 scope of this analysis.

In monocytes, miR-1291-5p emerged as a master miRNA regulator of the highest

368 number of genes associated with sPTL, including ILIB and its receptor ILIRI, which

369 have previously been associated with sPTL. The positive regulation of interleukin 2 gene

370 set was significantly enriched for miRNA target genes in both whole blood and

371 monocytes, which contains $I L 1 B$. This gene set is involved in immune response and

372 activation of inflammatory cytokines [31]. Inflammatory cytokines increase at the end of 
373 pregnancy as monocytes shift into an inflammatory M1 phenotype [30], and are

374 implicated in sPTL[32]. Our work suggests that this may be in part modulated through

375 changes in miRNAs expression in whole blood and monocytes.

We are limited in our ability to perform extensive characterization,

377 stratification/clustering or any predictive analyses of these miRNAs due to inadequate

378 statistical power resulting from a small sample size. Additionally, we cannot separate

379 miRNA signals related to PTL from those related to the labor process (uterine

380 contractions) or to the betamethasone treatment that women delivering prematurely

381 received prior to labor, as previously discussed [18] . Due this these limitations, this work

382 was not designed to interrogate miRNAs as a potential biomarker for sPTL. Further

383 functional analyses are required, including functional validation of miRNA/mRNA

384 targets, as well as the functionality of these miRNAs on key tissues involved in

385 pregnancy (i.e. placenta and myometrium). Additionally, our findings will need to be

386 validated in independent cohort of pregnant women. This work instead focuses on

387 potential mechanisms by which miRNAs may play a role in mediating systemic

388 inflammatory response in pregnant women that deliver prematurely, serves as an

389 important foundation to subsequent analyses, and highlights the importance of sample

390 source when performing blood related assessments.

Our analysis stands out among related studies because it is the first to generate

392 high throughput RNA sequencing data of both miRNA and mRNA from the same patient

393 source. We have carefully selected a reliable methodology to identify potential miRNA

394 targets based on experimental data [23], and have used novel alignment tools to obtain

395 comprehensive coverage of miRNA expression [20]. This robust, multi-dimensional data 
396 has allowed us to confidently build mRNA-miRNA regulation networks related to sPTL

397 in a way that has not previously been done, which provides additional insight into

398 mechanisms of transcriptional regulation of human labor. This type of integrative

399 analysis has been suggested to overcome many challenges in performing pregnancy

400 research [3]. Our analysis has highlighted miRNA mediated transcriptional regulatory

401 networks of sPTL-associated genes in monocytes and whole blood, which are involved in

402 important biological pathways, including interleukin signaling. Further work is needed to

403 validate these data in independent populations and elucidate the miRNA mechanisms of

404 signaling in whole blood and in a subpopulation of peripheral monocytes.

\section{ACKNOWLEDGEMENTS}

406 The authors would like to thank the women who participated in this study as well as the 407 staff at Mount Sinai Hospital, in particular Drs Robin Thurman and Kellie Murphy. We

408 would like to thank members of the Price lab, particularly Ben Heavner and Rory

409 Donovan for their help with the data management and members of Lye lab, particularly

410 Mrs Elzbieta Matysiak-Zablocki and Mrs Anna Dorogin for their help with blood

411 processing. We would like to acknowledge Drs Lee Hood, Stephen Matthews, Sam

412 Mesiano, Leslie Myatt, Craig Pennell and Yoel Sadovsky on behalf of the GAPPS

413 "Systems Biology of Preterm Birth" pilot study, as well as Mary Brunkow for her

414 coordination of this study. We would also like to thank Beverly Anne Bessie and Theresa

415 Fitzgerald for their help with the coordination of this manuscript. This work is supported

416 by the Global Alliance to Prevent Prematurity and Stillbirth (GAPPS), Grant Title

417 "Systems Biology of Preterm Birth: A Pilot Study", as well as the Eunice Kennedy 
Shriver National Institute of Child Health \& Human Development of the National

Institutes of Health: Grant R01HD091527 (to N.P), and K99HD096112 (to A.P).

\section{CONFLICTS OF INTEREST}

The authors confirm that there are no conflicts of interest.

[1] Blencowe H, Cousens S, Oestergaard MZ, et al. National, regional, and worldwide estimates of preterm birth rates in the year 2010 with time trends since 1990 for selected countries: a systematic analysis and implications. The Lancet n.d.; 379; 2162-72.

[2] Eidem HR, Ackerman WE, McGary KL, et al. Gestational tissue transcriptomics in term and preterm human pregnancies: a systematic review and meta-analysis. BMC Med. Genomics 2015; 8; 27.

[3] Eidem HR, McGary KL, Capra JA, et al. The transformative potential of an integrative approach to pregnancy. Placenta 2017; 57; 204-15.

[4] Tang M-X, Hu X-H, Liu Z-Z, et al. What are the roles of macrophages and monocytes in human pregnancy? J. Reprod. Immunol. 2015; 112; 73-80.

[5] Brown MB, von Chamier M, Allam AB, et al. M1/M2 Macrophage Polarity in Normal and Complicated Pregnancy. Front. Immunol. 2014; 5; 606.

[6] Hu Y, Li P, Hao S, et al. Differential expression of microRNAs in the placentae of Chinese patients with severe pre-eclampsia. Clin. Chem. Lab. Med. 2009; 47; 9239.

[7] Enquobahrie DA, Abetew DF, Sorensen TK, et al. Placental microRNA expression in pregnancies complicated by preeclampsia. Am. J. Obstet. Gynecol. 2011; 204; 178.e12-178.e21.

[8] Mayor-Lynn K, Toloubeydokhti T, Cruz AC, et al. Expression Profile of MicroRNAs and mRNAs in Human Placentas From Pregnancies Complicated by Preeclampsia and Preterm Labor. Reprod. Sci. 2011; 18; 46-56.

[9] Williams Z, Ben-Dov IZ, Elias R, et al. Comprehensive profiling of circulating microRNA via small RNA sequencing of cDNA libraries reveals biomarker potential and limitations. Proc. Natl. Acad. Sci. 2013; 110; 4255-60.

[10] Chim SSC, Lee WS, Ting YH, et al. Systematic identification of spontaneous preterm birth-associated RNA transcripts in maternal plasma. PloS One 2012; 7; e34328.

[11] Weber JA, Baxter DH, Zhang S, et al. The microRNA spectrum in 12 body fluids. Clin. Chem. 2010; 56; 1733-41.

[12] Chim SSC, Shing TKF, Hung ECW, et al. Detection and Characterization of Placental MicroRNAs in Maternal Plasma. Clin. Chem. 2008; 54; 482-90.

[13] Gunel T, Zeybek YG, Akcakaya P, et al. Serum microRNA expression in pregnancies with preeclampsia. Genet. Mol. Res. GMR 2011; 10; 4034-40. 
[14] Gray C, McCowan LM, Patel R, et al. Maternal plasma miRNAs as biomarkers during mid-pregnancy to predict later spontaneous preterm birth: a pilot study. Sci. Rep. 2017; 7; 815.

[15] Wommack JC, Trzeciakowski JP, Miranda RC, et al. Micro RNA clusters in maternal plasma are associated with preterm birth and infant outcomes. PLOS ONE 2018; 13; e0199029.

[16] Whitehead CL, Teh WT, Walker SP, et al. Circulating MicroRNAs in Maternal Blood as Potential Biomarkers for Fetal Hypoxia In-Utero. PLoS ONE 2013; 8; e78487.

[17] Dang T-M, Wong W-C, Ong S-M, et al. MicroRNA expression profiling of human blood monocyte subsets highlights functional differences. Immunology 2015; $145 ; 404-16$.

[18] Paquette AG, Shynlova O, Kibschull M, et al. Comparative analysis of gene expression in maternal peripheral blood and monocytes during spontaneous preterm labor. Am. J. Obstet. Gynecol. 2018; 218; 345.e1-345.e30.

[19] Fallen S, Baxter D, Wu X, et al. Extracellular vesicle RNAs reflect placenta dysfunction and are a biomarker source for preterm labour. J. Cell. Mol. Med. 2018; DOI: $10.1111 /$ jcmm.13570.

[20] Wu X, Kim T-K, Baxter D, et al. sRNAnalyzer-a flexible and customizable small RNA sequencing data analysis pipeline. Nucleic Acids Res. 2017; DOI: 10.1093/nar/gkx999.

[21] Griffiths-Jones S, Saini HK, van Dongen S, et al. miRBase: tools for microRNA genomics. Nucleic Acids Res. 2008; 36; D154-8.

[22] Robinson MD, McCarthy DJ, Smyth GK. edgeR: a Bioconductor package for differential expression analysis of digital gene expression data. Bioinformatics 2010; $26 ; 139-40$.

[23] Agarwal V, Bell GW, Nam J-W, et al. Predicting effective microRNA target sites in mammalian mRNAs. ELife 2015; 4; e05005.

[24] Shannon P, Markiel A, Ozier O, et al. Cytoscape: a software environment for integrated models of biomolecular interaction networks. Genome Res. 2003; 13; 2498-504.

[25] Seitz H, Royo H, Bortolin M-L, et al. A large imprinted microRNA gene cluster at the mouse Dlk1-Gt12 domain. Genome Res. 2004; 14; 1741-8.

[26] Paquette A, Chu, Tianjiao, Wu, Xiaogang, et al. Distinct communication patterns of trophoblastic miRNA among the maternal-placental-fetal compartments. Placenta n.d.; 72-73; 28-35.

[27] Allis CD, Jenuwein T. The molecular hallmarks of epigenetic control. Nat. Rev. Genet. 2016; 17; 487.

[28] Romero R, Dey SK, Fisher SJ. Preterm labor: one syndrome, many causes. Science 2014; 345; 760-5.

[29] Ball G, Srinivasan L, Aljabar P, et al. Development of cortical microstructure in the preterm human brain. Proc. Natl. Acad. Sci. U. S. A. 2013; 110; 9541-6.

[30] Zhang Y-H, He M, Wang Y, et al. Modulators of the Balance between M1 and M2 Macrophages during Pregnancy. Front. Immunol. 2017; 8; 120.

[31] Hoyer KK, Dooms H, Barron L, et al. Interleukin-2 in the development and control of inflammatory disease. Immunol. Rev. 2008; 226; 19-28. 
503

504

505

506
[32] Nadeau-Vallee M, Obari D, Quiniou C, et al. A critical role of interleukin-1 in preterm labor. Cytokine Growth Factor Rev. 2016; 28; 37-51.

\section{TABLES}

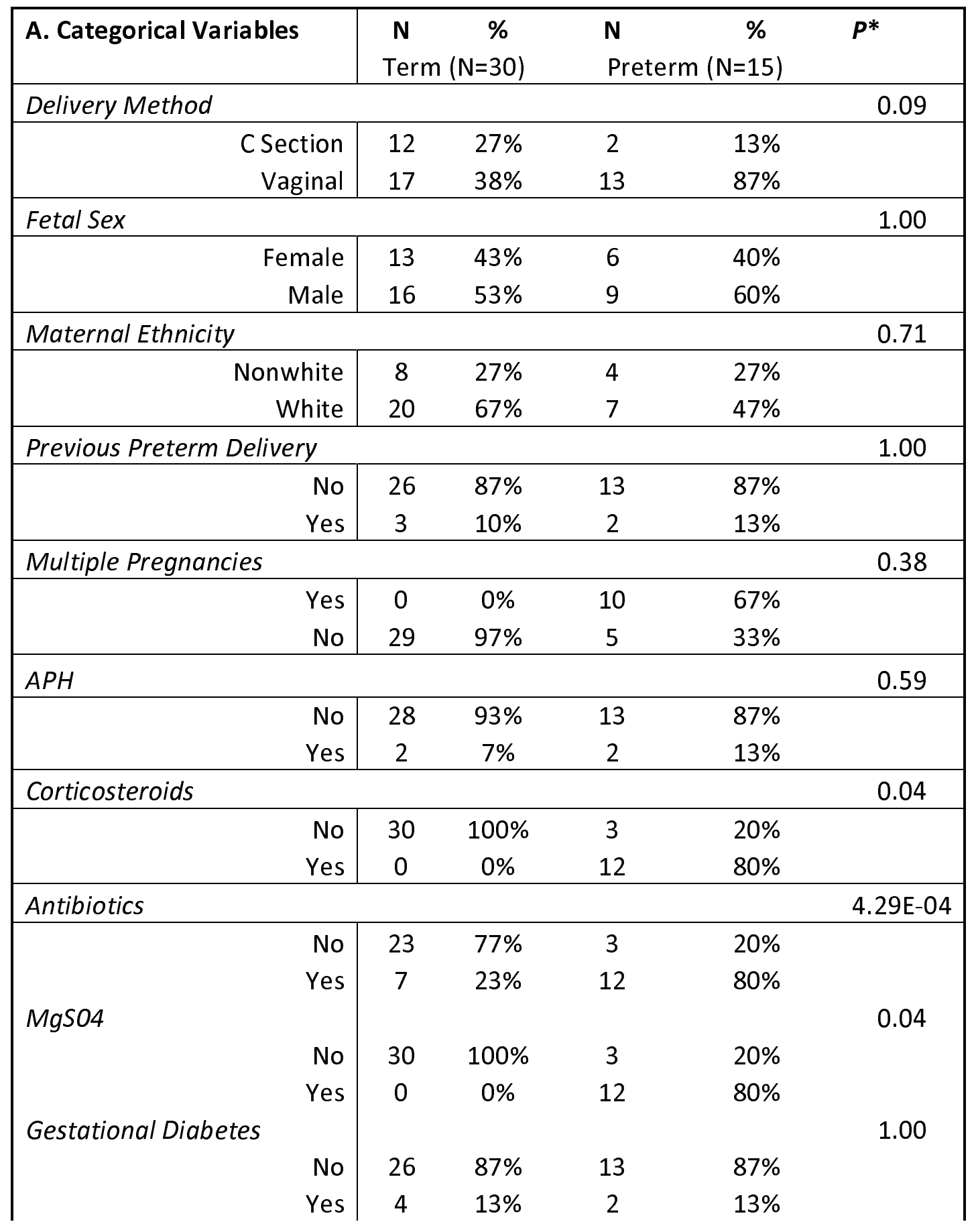




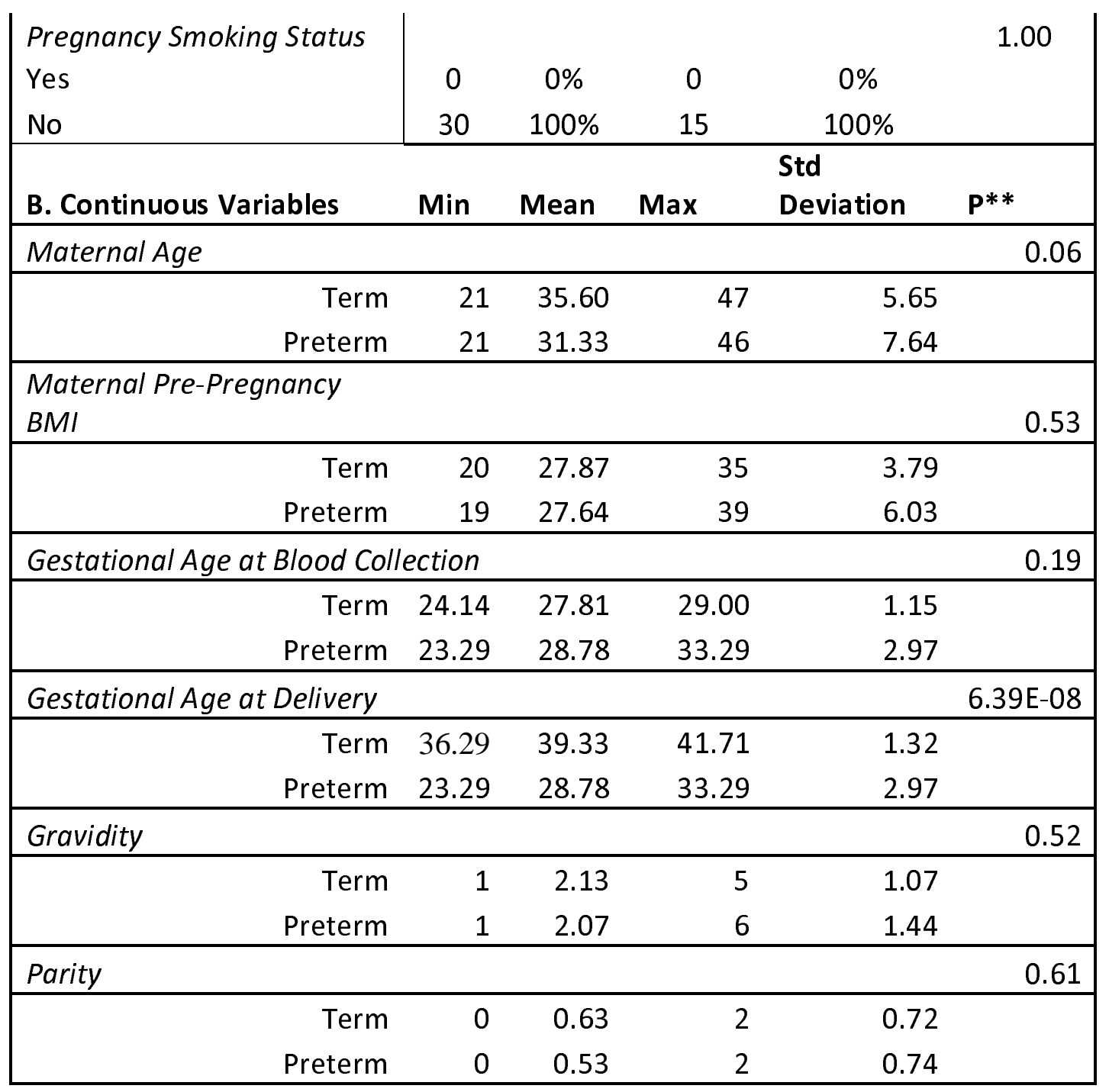

510 Table 2: mRNA targets of differentially expressed miRNAs involved in Positive

511 regulation of Interleukin-2 signaling

\begin{tabular}{|ll|}
\hline miRNA & Gene targets in GO:0032743 \\
\hline Monocytes & \\
\hline
\end{tabular}




\begin{tabular}{|c|c|}
\hline hsa-miR-1248-5p & SASH3, CD83 \\
\hline hsa-miR-1291-5p & $I L 1 B$ \\
\hline hsa-miR-378c-5p & CD28,TNFSF4 \\
\hline \multicolumn{2}{|l|}{ Whole Blood } \\
\hline hsa-miR-4742-3p & CCR2 \\
\hline hsa-miR-1299-3p & $C D 4, M A L T 1$ \\
\hline hsa-miR-582-3p & $P D E 4 B$ \\
\hline hsa-miR-1291-5p & CD28 \\
\hline
\end{tabular}

Figure 1: MA plot of $\log 2$ fold changes vs. the mean of normalized counts in the regularized logarithmic distribution of RNA sequencing data in (A) monocytes, and (C)

518 whole blood. mRNAs significantly different in linear models after adjustment for 519 multiple comparisons are highlighted in red, and lines indicate $\log 2$ fold changes $>1$.

520 Heatmap of the 16 differentially expressed genes identified in monocytes $(\mathbf{B})$ or the 9 521 differentially expressed genes identified in whole blood (D), with blue indicating lower 522 expression and red indicating higher expression. miRNA expression of women who 523 underwent term labor is on the left and highlighted in grey, and miRNA expression of 524 women in SPTL is highlighted in blue on the right.

525 Figure 2: A. Venn diagram in whole blood (A) and monocytes (B) of genes previously 526 associated with SPTL and gene targets of the differentially expressed miRNAs identified 527 in this study. In the overlapping genes, we show the log fold change and $\mathrm{P}$ values of the 
528 miRNAs (from supplemental table 1), correlation with mRNA concentration, and log fold

529 change and correlation with sPTL in the previous study).

530 Figure 3: Biomolecular network of miRNA target genes in (A) monocytes and (B) whole

531 blood. Enrichment analysis was performed on gene ontology (GO) gene sets and

532 significant gene sets are shown (Benjamini Hoch adjusted $\mathrm{q}<0.05$ ). Network node layout

533 is based on similarity between genes within GO gene sets, where nodes are colored based

534 on multiple occurrences within different go categories. GO terms which were significant

535 in both whole blood and monocytes have a red outer circle.

536 Supplemental Figure 1: miR-1291-5p expression in whole blood and monocytes.

537 miRNA expression values for women who delivered at term are highlighted in orange,

538 and values for women who delivered prematurely are in blue.

539 Supplemental Figure 2: Correlations in miRNA expression which were identified as

540 significant in (A) monocytes and plasma (B) monocytes and exosomes (C) whole blood

541 and plasma/exosomes. Blue dots indicate correlations involving monocytes, and red dots

542 indicate correlations involving whole blood. Orange dots reflect correlation between

543 whole blood and exosomes. Circled dots are expression values involving exosomes.

544 Correlations were evaluated using spearman correlations, with significance defined as

$545 p<0.05$. All correlations; including those which are insignificant are shown here.

546 Supplemental Figure 3: Number of miRNA targets identified in (A) whole blood and

547 (B) Monocytes. Blue lines indicates the number of targetscan candidates which had

548 higher than the median context ++ scores, which is a measure of prediction accuracy to

549 bind to target miRNA. Green bars indicate mRNAs with matched expression data in 
550 GSE96097. Yellow bars indicate samples whose miRNA expression in whole blood

551 and/or monocytes was significantly correlated with the mRNA expression (defined as $\rho$

$552<-0.3, p<0.05$, spearman correlation coefficients) in matched samples. 
A. miRNA target genes associated with SPTL: Whole Blood

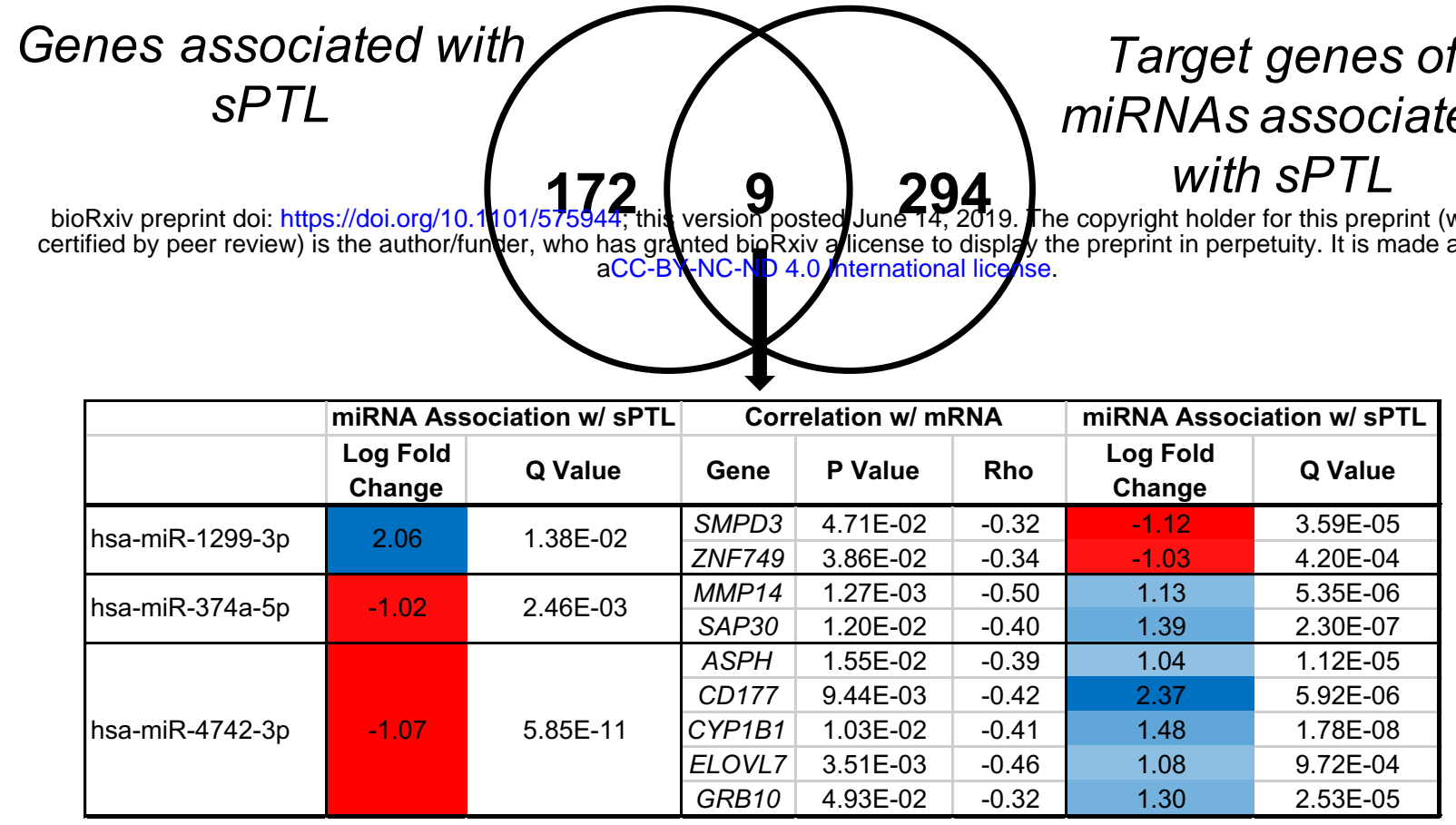

\section{B. miRNA target genes associated with sPTL: Monocytes}

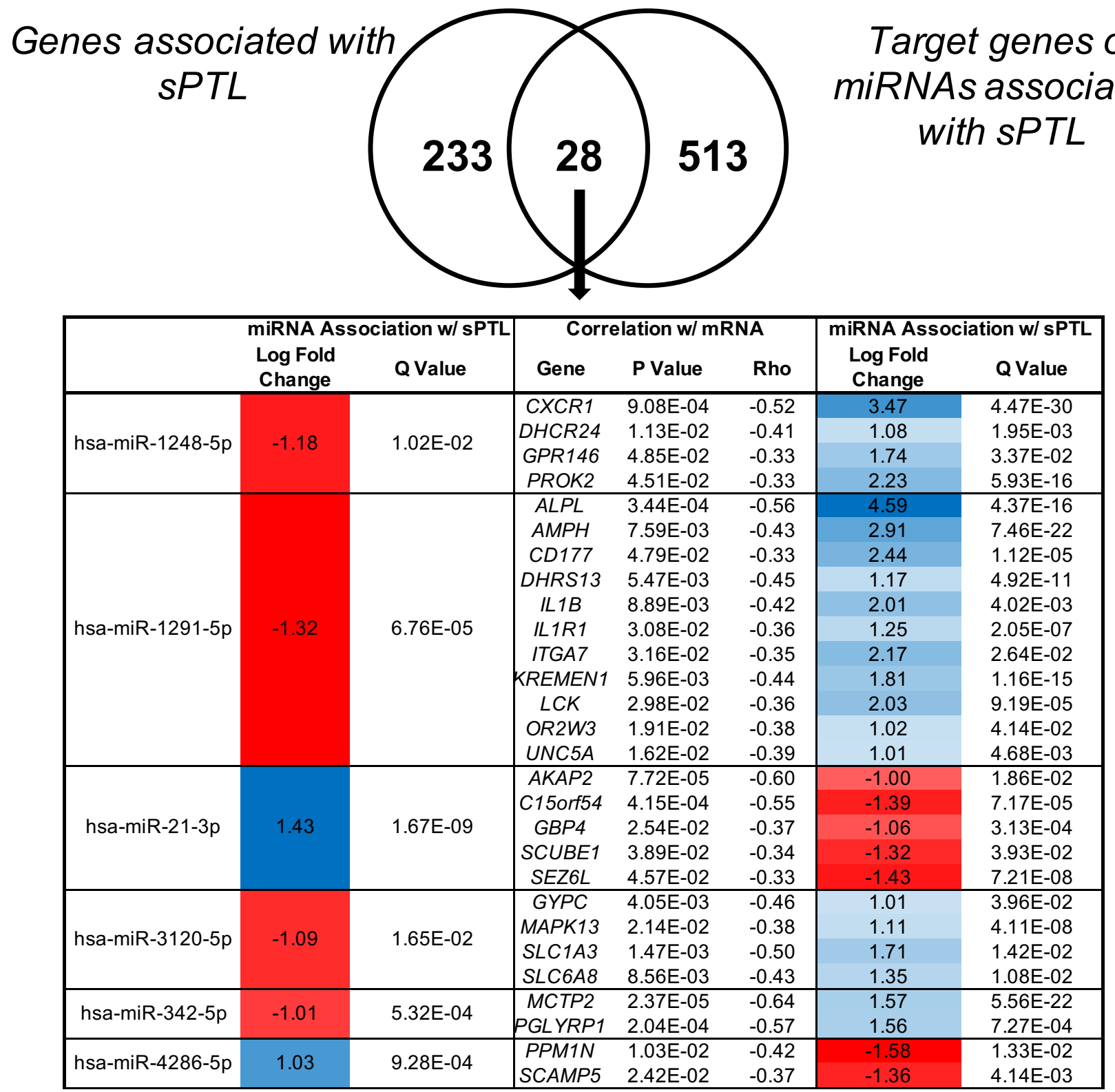




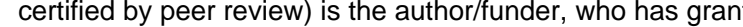

\section{A. Pathway Enrichment: miRKNA target Gennes (Whole Blood)}

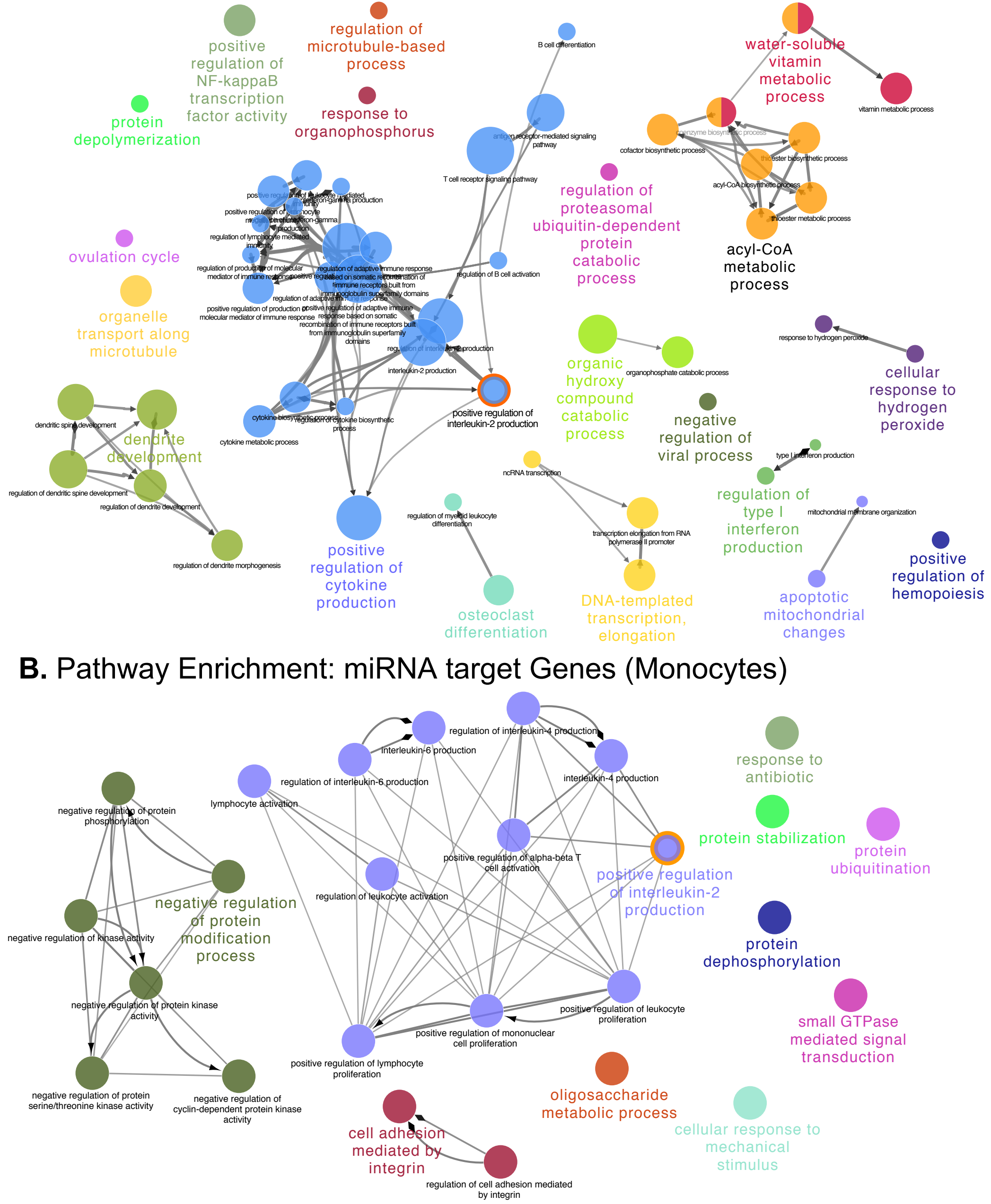

